\title{
Independent sets in $\left(P_{6}\right.$, diamond)-free graphs
}

\author{
Raffaele Mosca \\ Dipartimento di Scienze, Universitá degli Studi ”G. d'Annunzio”, Pescara 65127, Italy. \\ r.mosca@unich.it
}

received June 6, 2006, revised June 6, 2007, March 17, 2008, January 23, 2009, accepted January 27, 2009.

We prove that on the class of $\left(P_{6}\right.$,diamond)-free graphs the Maximum-Weight Independent Set problem and the Minimum-Weight Independent Dominating Set problem can be solved in polynomial time.

Keywords: Maximum-Weight Independent Set; Minimum-Weight Independent Dominating Set; polynomial-time algorithm.

\section{Introduction}

An independent set (or a stable set) in a graph $G$ is a subset of pairwise nonadjacent vertices of $G$. An independent set of $G$ is maximal if it is not properly contained in any other independent set of $G$.

The Maximum-Weight Independent Set (WIS) problem is the following: Given a graph $G=(V, E)$ and a weight function $w$ on $V$, determine an independent set of $G$ of maximum weight. Let $\alpha_{w}(G)$ denote the maximum weight of an independent set of $G$. The WIS problem reduces to the $I S$ problem if all vertices $v$ have the same weight $w(v)=1$.

The WIS problem is NP-hard [34] and remains difficult for cubic graphs [27] and for planar graphs [26], while it can be efficiently solved for various graph classes which include perfect graphs [33] (and the class of perfect graphs includes the chordal graphs), $K_{1,3}$-free graphs [2, 37, 40, 42, 45], and $2 K_{2}$-free graphs [21, 22, 38].

The Minimum-Weight Independent Dominating Set (WID) problem is the following: Given a graph $G=(V, E)$ and a weight function $w$ on $V$, determine a maximal independent set of $G$ of minimum weight. Let $\iota_{w}(G)$ denote the minimum weight of a maximal independent set of $G$. The WID problem reduces to the $I D$ problem if all vertices $v$ have the same weight $w(v)=1$.

The WID problem is NP-hard [28] and remains difficult for chordal graphs [18] and for $2 P_{3}$-free perfect graphs [46], while it can be efficiently solved for various graph classes which include permutation graphs [15], locally independent well-dominated graphs [47], and $2 K_{2}$-free graphs [21, 22, 38].

Both WIS and WID remain difficult for triangle-free graphs [43]. Also, for both IS and ID, the class of $P_{5}$-free graphs is the unique minimal class, defined by forbidding a single connected subgraph, for which the computational complexity is an open question (see [1, 3, 7]).

On the other hand, several papers introduced structural properties on graphs containing no long induced paths (see e.g. [5, 6, 19, 39]), often applied to design efficient algorithms for solving various NP-hard 
problems, including WIS or WID, in subclasses of such graphs: concerning subclasses of $P_{5}$-free graphs, see e.g. [4, 9, 10, 11, 13, 16, 17, 23, 29, 31, 36]; concerning subclasses of $P_{6}$-free graphs, see e.g. [9, 24, 30, 35, 41, 44].

Let us focus on two such graph subclasses which involve triangle-free graphs as well.

The class of $\left(P_{5}\right.$,diamond)-free graphs: a recent paper [8] shows that such graphs have bounded cliquewidth and that a corresponding clique-width expression can be constructed in $\mathcal{O}(n+m)$ time, which implies that a large class of NP-hard problems including WIS and WID can be solved for such graphs in $\mathcal{O}(n+m)$ time.

The class of $\left(P_{6}\right.$,triangle)-free graphs: a recent paper [14] shows that such graphs have bounded cliquewidth and that a corresponding clique-width expression can be constructed in $\mathcal{O}\left(n^{2}\right)$ time, which implies that a large class of NP-hard problems including WIS and WID can be solved for such graphs in $\mathcal{O}\left(n^{2}\right)$ time.

This paper introduces a proof that WIS and WID can be solved for ( $P_{6}$,diamond)-free graphs in $\mathcal{O}\left(n^{7}\right)$ time.

\section{Notation and preliminaries}

For any missing notation or reference, let us refer to [12].

Throughout this paper let $G=(V, E)$ be a finite undirected graph without self-loops and multiple edges and let $|V|=n,|E|=m$. For every $u \in V$, let $N(u)=\{v \in V: u v \in E\}$ be the set of neighbors of $u$. Let $U, W$ be two subsets of $V$. Let $N(U)=\{v \in V \backslash U$ : there exists $u \in U$ such that $u v \in E\}$. Let $N_{W}(U)=N(U) \cap W$. Let us say that $U$ has a join (a co-join, respectively) with $W$, if each vertex in $U$ is adjacent (is nonadjacent) to each vertex in $W$. Let $v \in V$. Let us say that: $v$ contacts $U$ if $v$ is adjacent to some vertex of $U ; v$ is universal for $U$ if $v$ is adjacent to each vertex of $U ; v$ is partial to $U$ if $v$ contacts $U$ but is not universal for $U$. Then let us say that $U$, with $\emptyset \subset U \subset V$, is a module of $G-$ often called homogeneous set in the literature - if no vertex of $V \backslash U$ is partial to $U$.

Let $G[U]$ denote the subgraph of $G$ induced by the vertex subset $U$. For any graph $F, G$ is $F$-free if $G$ contains no induced subgraph isomorphic to $F$.

A component of $G$ is the vertex set of a maximal connected subgraph of $G$. A component of $G$ is trivial if it is a singleton, and nontrivial otherwise.

Concerning WIS and WID, algorithmically an easy reduction works if the graph is disconnected: that is, if $G$ has components $V_{1}, \ldots, V_{k}$, then $\alpha_{w}(G)=\alpha_{w}\left(G\left[V_{1}\right]\right)+\ldots+\alpha_{w}\left(G\left[V_{k}\right]\right)$ and $\iota_{w}(G)=\iota_{w}\left(G\left[V_{1}\right]\right)+$ $\ldots+\iota_{w}\left(G\left[V_{k}\right]\right)$.

A path $P_{k}$ has vertices $v_{1}, v_{2}, \ldots, v_{k}$ and edges $v_{j} v_{j+1}$ for $1 \leq j<k$. A cycle $C_{k}$ has vertices $v_{1}, v_{2}, \ldots, v_{k}$ and edges $v_{j} v_{j+1}$ for $1 \leq j \leq k$ (subscript addition taken modulo $k$ ). A triangle is a graph of three vertices $a, b, c$ and edges $a b, a c, b c$. A diamond is a graph of four vertices $a, b, c, d$ and edges $a b, a c, a d, b c, b d$.

A clique of $G$ is a set of pairwise adjacent vertices of $G$. Notice that each component of $G$ is a clique if and only if $G$ is $P_{3}$-free.

A graph is chordal if it contains no induced $C_{k}, k \geq 4$.

For chordal graphs, WIS and ID can be efficiently solved (see [25] and [20], respectively), while WID remains NP-hard on them [18].

In [32] the authors proved that distance-hereditary graphs have bounded clique-width, and that a corresponding clique-width expression can be constructed in $\mathcal{O}(n+m)$ time. Since chordal diamond-free 
graphs are distance-hereditary, a direct consequence is:

Theorem 1 ([32]) Both the WIS and the WID problems can be solved for chordal diamond-free graphs in $\mathcal{O}(n+m)$ time.

In [14] the authors proved that ( $P_{6}$,triangle)-free graphs have bounded clique-width, and that a corresponding clique-width expression can be constructed in $\mathcal{O}\left(n^{2}\right)$ time. A direct consequence is:

Theorem 2 ([14]) Both the WIS and the WID problems can be solved for $\left(P_{6}\right.$,triangle)-free graphs in $\mathcal{O}\left(n^{2}\right)$ time.

Obviously, the WIS (or WID) problem on a graph $G$ with vertex weight function $w$ can be reduced to the same problem on subgraphs $G[V \backslash N(v)]$ for every $v \in V$ in the following way:

$$
\begin{gathered}
\alpha_{w}(G)=\max \left\{\alpha_{w}(G[V \backslash N(v)]) \mid v \in V\right\} \\
\iota_{w}(G)=\min \left\{\iota_{w}(G[V \backslash N(v)]) \mid v \in V\right\}
\end{gathered}
$$

Thus, whenever WIS (or WID) is solvable in time $T$ for every subgraph $G[V \backslash N(v)]$ of $G$ with $v \in V$, then it is solvable for $G$ in time $n T$, plus $\mathcal{O}\left(n^{3}\right)$ additional steps to generate those subgraphs.

Let us conclude with an observation which will be often used later.

Observation 1 Let $G=(V, E)$ be a graph, and $U \subseteq V$ with $|U|=k$. If one can solve WIS (or WID) for each induced subgraph of $G[V \backslash U]$ in time $T$, then one can solve WIS (or WID) for $G$ in time $2^{k}\left(T+n^{2}\right)$.

Proof: Let $I(U)$ be the family of independent sets of $G[U]$. Then to solve WIS (or WID) for $G$ one can consider WIS (or WID) for $|I(U)|$ subgraphs of $G$, i.e., for $G[V \backslash U]$ and for $G[I \cup(V \backslash(N(I) \cup U))]$ for every $I \in I(U)$. Since $|I(U)| \leq 2^{k}$, the assertion follows.

Remark: The results of the next section are introduced only for WIS, by meaning that they hold for WID as well (by interchanging WIS with WID, and $\alpha$ with $\iota$ ).

\section{Independent sets in ( $P_{6}$, diamond)-free graphs}

Let us introduce a preliminary result.

Definition 1 A graph $G=(V, E)$ is green if there exists a partition $\{X, Y\}$ of $V$ (with $X$ or $Y$ possibly empty) such that:

(i) $G[X]$ and $G[Y]$ are $P_{3}$-free;

(ii) each component of $G[Y]$ is a module of $G$;

(iii) each vertex in $Y$ is adjacent to at most one vertex in each component of $G[X]$.

Notice that every $P_{3}$-free graph is green.

Lemma 1 One can solve WIS for every green $P_{6}$-free graph in $O\left(n^{3}\right)$ time. 
Proof: Let $G=(V, E)$ be a green $P_{6}$-free graph. Assume without loss of generality that $G$ is connected. Let $\{X, Y\}$ be a partition of $V$ according to Definition 1. In particular, to our aim, by iil one can assume that $Y$ is an independent set. In fact, one can contract each component $K$ of $G[Y]$ into a singleton $u$ with $w(u)=\alpha_{w}(G[K])$ : that can be done in polynomial time since each component of $G[Y]$ is a clique.

Let $W$ be the family of nontrivial components of $G[X]$. In particular, to our aim (similarly to above), one can assume that in each component $Q \in W$ at most one vertex is nonadjacent to any vertex in $Y$.

Claim 3.1 There exists $y^{*} \in Y$ such that $y^{*}$ contacts every element of $W$.

Proof: For any $y \in Y$, let $J(y)=\{Q \in W: y$ contacts $Q\}$. Let $y^{*} \in Y$ be such that $\left|J\left(y^{*}\right)\right| \geq|J(y)|$ for every $y \in Y$. We state that this vertex $y^{*}$ is a proper choice for Claim 3.1. Assume for a contradiction that there exists a component $Q \in W$ such that $y^{*}$ does not contact $Q$. Since $G$ is connected, one can select $y \in Y$ belonging to a shortest path from $y^{*}$ to $Q$, such that $y$ contacts $Q$. By definition of $W$ and by iiii, $Q$ contains two (adjacent) vertices $q_{1}$ and $q_{2}$ such that $y$ is adjacent to $q_{1}$ and nonadjacent to $q_{2}$. Then, since $G$ is $P_{6}$-free, $y$ contacts all the elements of $W$ which are contacted by $y^{*}$. This implies $\left|J\left(y^{*}\right)\right|<|J(y)|$, a contradiction.

Claim 3.2 There exists at most one element of $W$ of cardinality greater than 2.

Proof: Assume for a contradiction that there exist two elements of $W$, say $\tilde{Q}$ and $Q$, with $|\tilde{Q}| \geq 3$ and $|Q| \geq 3$. Let $y^{*} \in Y$ according to Claim 3.1 Let $\tilde{q} \in \tilde{Q}$ and $q \in Q$ be adjacent to $y^{*}$. Since $|\tilde{Q}| \geq 3$, there exists $a \in Y$ adjacent to $q_{a} \in \tilde{Q}$, with $q_{a} \neq \tilde{q}$. Then $a$ is adjacent to $q$, otherwise $a, q_{a}, \tilde{q}, y^{*}, q$ and any vertex in $Q$ nonadjacent to $a$ induce a $P_{6}$. Then, since $|Q| \geq 3$, there exists $b \in Y$ adjacent to $q_{b} \in Q$, with $q_{b} \neq q$. By symmetry, one has that $b$ is adjacent to $\tilde{q}$. Since $|Q| \geq 3$, there exists $q^{\prime} \in Q$ such that $q^{\prime} \neq q$ and $q^{\prime}$ is nonadjacent to $b$. Then $q^{\prime}, q_{b}, b, \tilde{q}, q_{a}, a$ induce a $P_{6}$, a contradiction.

If $|Q| \leq 2$ for every $Q \in W$, then by iii $G$ is triangle-free. Otherwise, by Claim 3.2 there exists at most one element, say $\tilde{Q}$, of $W$ of cardinality greater than 2. One can solve WIS in $G$ by solving WIS in $G[V \backslash \tilde{Q}]$ and in $G[V \backslash N(\tilde{q})]$ for every $\tilde{q} \in \tilde{Q}$. Since such graphs are triangle-free, the lemma follows by Theorem 2

\subsection{Deleting $C_{6}$ 's in ( $P_{6}$, diamond)-free graphs}

Throughout this subsection assume that $G=(V, E)$ is a ( $P_{6}$,diamond)-free graph containing a 6-cycle $C$, say with vertices $v_{i}$ and edges $v_{i} v_{i+1}, i \in\{1, \ldots, 6\}$ (subscript addition taken modulo 6). Let $N(C)$ be the set of vertices from $V \backslash C$ which are adjacent to some vertex in $C$. For any subset $S$ of $C$, let $M_{S}$ be the set formed by those vertices in $N(C)$ which are adjacent to each vertex in $S$ and are nonadjacent to each vertex in $C \backslash S$. In particular, let us write $M_{1}$ for $S=\left\{v_{1}\right\}, M_{1,2}$ for $S=\left\{v_{1}, v_{2}\right\}$, and so on. Then let $Z(k)$ denote the set of vertices of $N(C)$ having exactly $k$ neighbors in $C$.

Since $G$ is ( $P_{6}$, diamond)-free: $Z(1)=Z(5)=Z(6)=\emptyset$; each vertex in $Z(2)$ belongs to some of the sets $M_{i, i+2}$ or $M_{i, i+3}, i \in\{1, \ldots, 6\}$ (subscript addition taken modulo 6); each vertex in $Z$ (3) belongs to some of the sets $M_{i, i+2, i+4}$ or $M_{i, i+2, i+3}$ or $M_{i, i+3, i+4}, i \in\{1, \ldots, 6\}$ (subscript addition taken modulo 6).

Lemma 2 Every component of $G[Z(0)]$ is green. 
Proof: Let $K$ be a component of $G[Z(0)]$. Since $G$ is connected, there exists $v \in V \backslash K$ which contacts $K$. If $v$ is universal for $K$, then $G[K]$ is $P_{3}$-free (since $G$ is diamond-free). Then let us assume that $v$ is partial to $K$, and prove that $G[K]$ is green. Let us write $X=K \cap N(v)$, and $Y=K \backslash N(v)$. Since $G$ is diamond-free, $G[X]$ is $P_{3}$-free. Let $T$ be a component of $G[Y]$. Then $T$ is a module of $G[K]$ : otherwise, for any $x \in X$ partial to $T$, one has that two adjacent vertices in $T$ together with $x, v$ and two vertices in $C$ would induce a $P_{6}$ (since $G$ is $\left(P_{6}\right.$,diamond)-free, $v$ is the endpoint of an induced $P_{3}$ involving two vertices in $C$ ). Then $T$ is a clique (since $G$ is diamond-free), i.e., $G[Y]$ is $P_{3}$-free. Furthermore, each vertex in $Y$ is adjacent to at most one vertex in each component of $X$, otherwise a diamond arises involving $v$. Then the lemma follows.

Let us fix any vertex of $C$, say $v_{2}$.

Let us prove that WIS can be solved for $G\left[V \backslash N\left(v_{2}\right)\right]$ in $\mathcal{O}\left(n^{6}\right)$ time.

A partition of $V \backslash N\left(v_{2}\right)$ is given by $\left\{\left\{v_{2}, v_{4}, v_{5}, v_{6}\right\}, M_{1,3,4,6}, M_{1,3,5}, M_{1,3,4}, M_{1,4,5}\right.$,

$\left.M_{3,5,6}, M_{3,6,1}, M_{4,6,1}, M_{6,3,4}, M_{1,3}, M_{1,4}, M_{1,5}, M_{3,5}, M_{3,6}, M_{4,6}, Z(0)\right\}$.

Since $G$ is diamond-free, the sets $M_{1,3,4,6}, M_{1,3,4}, M_{1,4,5}, M_{3,5,6}, M_{3,6,1}, M_{4,6,1}, M_{6,3,4}$ have cardinality at most one. Then, by Observation 1 , to our aim it is sufficient to prove that WIS can be solved for each induced subgraph of $G[U]$ in polynomial time, where a partition of $U$ is given by

$\left\{M_{1,3,5}, M_{1,3}, M_{1,4}, M_{1,5}, M_{3,5}, M_{3,6}, M_{4,6}, Z(0)\right\}$.

Since $G$ is diamond-free: $M_{1,3,5}, M_{1,3}, M_{1,4}, M_{1,5}, M_{3,5}, M_{3,6}, M_{4,6}$ are independent sets. Since $G$ is ( $P_{6}$, diamond)-free: $M_{1,3,5} \cup M_{1,3} \cup M_{1,5} \cup M_{3,5}$ is an independent set; $M_{1,3} \cup M_{1,5} \cup M_{3,5} \cup M_{4,6}$ has a co-join with $Z(0)$.

For any $W \subseteq U$, let us write $W^{*}=W \cap Z(0)$.

For any $W \subseteq U$, let us say that a component $K$ of $G\left[W^{*}\right]$ is of:

type 1 if $K$ is not a clique and there exists a vertex in $W \backslash Z(0)$ partial to $K$;

type 2 if $K$ is a clique and there exists a vertex in $W \backslash Z(0)$ partial to $K$;

type 3 otherwise.

Let $T_{1}(W), T_{2}(W), T_{3}(W)$ respectively denote the union of components of $G\left[W^{*}\right]$ of type $1,2,3$.

Let us fix a subset $W \subseteq U$.

Notice that $M_{1,3,5}$ is an independent set, since $G$ is diamond-free.

Let us consider the following binary relation ' $\leq_{1}$ ' on $M_{1,3,5}$ : for any $a, b \in M_{1,3,5}, a \leq_{1} b$ if $N_{T_{1}(W)}(a) \subseteq$ $N_{T_{1}(W)}(b)$. It is immediate to verify that $\left(M_{1,3,5}, \leq_{1}\right)$ is a partially ordered set.

Lemma 3 Let $y \in M_{1,3,5}$ be maximal for $\left(M_{1,3,5}, \leq_{1}\right)$. Then $G\left[T_{1}(W) \backslash N(y)\right]$ is $P_{3}$-free.

Proof: First let us prove that $y$ contacts every component $Q$ of type 1 of $G\left[W^{*}\right]$. Assume for a contradiction that there exists a component $Q_{1}$ of type 1 of $G\left[W^{*}\right]$ such that $y$ does not contact $Q_{1}$. By definition of component of type 1, there exists $y_{1} \in W \backslash Z(0)$ partial to $Q_{1}$. Since $G$ is $P_{6}$-free (also recall that $W \subseteq U), y_{1} \in M_{1,3,5}$. By the maximality of $y$ there exists a vertex $\tilde{q}$ in some component $\tilde{Q}$ of type 1 of $G\left[W^{*}\right]$ such that $\tilde{q}$ is adjacent to $y$ and nonadjacent to $y_{1}$. Then $\tilde{q}, y, v_{1}, y_{1}$ and two adjacent vertices of $Q_{1}$ induce a $P_{6}$, a contradiction.

To conclude the proof of the lemma one has to show that if $y$ contacts a component $Q$ of type 1 of $G\left[W^{*}\right]$, then $G[Q \backslash N(y)]$ is $P_{3}$-free. This can be shown by applying the last part of the argument of Lemma2, 
Let us consider the following binary relation ' $\leq_{2}$ ' on $M_{1,3,5}$ : for any $a, b \in M_{1,3,5}, a \leq_{2} b$ if $N_{T_{2}(W)}(a) \subseteq N_{T_{2}(W)}(b)$. It is immediate to verify that $\left(M_{1,3,5}, \leq_{2}\right)$ is a partially ordered set.

Lemma 4 Let $y \in M_{1,3,5}$ be maximal for $\left(M_{1,3,5}, \leq_{2}\right)$. If $T_{1}(W)=\emptyset$, then $G\left[(W \backslash N(y))^{*}\right]$ admits at most one component of type 2.

Proof: Let $Q_{1}$ be a component of type 2 of $G\left[(W \backslash N(y))^{*}\right]$. By definition of component of type 2 there exists $y_{1} \in(W \backslash N(y)) \backslash Z(0)$ partial to $Q_{1}$. Since $G$ is $P_{6}$-free (also recall that $\left.W \subseteq U\right), y_{1} \in M_{1,3,5}$. Let $q_{1} \in Q_{1}$ be adjacent to $y_{1}$; let $q^{\prime} \in Q_{1}$ be nonadjacent to $y_{1}$. Since $T_{1}(W)=\emptyset$, there exists a component of type 2 of $G\left[W^{*}\right]$, say $\tilde{Q}_{1}$, such that $Q_{1} \subseteq \tilde{Q}_{1}$. In particular, there exists $\tilde{q}_{1} \in \tilde{Q}_{1} \backslash Q_{1}$ adjacent to $y$ and nonadjacent to $y_{1}$, otherwise, by the maximality of $y$ there would exist a vertex $t \in W \backslash \tilde{Q}_{1}$ adjacent to $y$ and nonadjacent to $y_{1}$, i.e., vertices $q^{\prime}, q_{1}, y_{1}, v_{1}, y, t$ would induce a $P_{6}$.

Let us prove that $Q_{1}$ is the unique component of type 2 of $G\left[(W \backslash N(y))^{*}\right]$. Assume for a contradiction that there exists another component of type 2, say $Q_{2}$, of $G\left[(W \backslash N(y))^{*}\right]$. Notice that $y_{1}$ is nonadjacent to any vertex $q_{2}$ of $Q_{2}$, otherwise $q^{\prime}, \tilde{q}_{1}, y, v_{1}, y_{1}$ and $q_{2}$ would induce a $P_{6}$. Then let $y_{2} \in M_{1,3,5}$ be partial to $Q_{2}$. By symmetry, $y_{2}$ is nonadjacent to any vertex of $Q_{1}$. Then a vertex of $Q_{2}, y_{2}, v_{1}, y_{1}, q_{1}, q^{\prime}$ induce a $P_{6}$, a contradiction.

Now, let us consider the following cases:

1. $T_{1}(U)=T_{2}(U)=\emptyset$.

Then each component $K$ of $G[Z(0)]$ is a module of $G[U]$. Then, to our aim, one can assume that $K$ is a singleton. In fact, one can contract $K$ into a singleton $u$ with $w(u)=\alpha_{w}(G[K])$ : that can be done in polynomial time by Lemmas 2 and 1 . So in general, one can assume that $Z(0)$ is an independent set. One can solve WIS in $G[U]$ by solving WIS in $G\left[U \backslash M_{1,4}\right]$ and in $G[U \backslash N(y)]$, for every $y \in M_{1,4}$.

That can be done in $\mathcal{O}\left(n^{3}\right)$ time. In fact, by the assumptions and by the above properties, one can verify that $G\left[U \backslash M_{1,4}\right]$ is triangle-free, and that $G[U \backslash N(y)]$ is triangle-free for every vertex $y \in M_{1,4}$ (in particular, no vertex of $M_{3,6} \backslash N(y)$ is adjacent to a vertex of $Z(0) \backslash N(y)$, otherwise a $P_{6}$ arises). Then the assertion follows by Theorem 2

For the other two cases we note that the existence of a component $Q$ of type 1 or type 2 in $U \cap Z(0)$ implies the existence of a vertex $a \in M_{1,3,5}$ which is partial to $Q$, similarly to the proof above. So, $M_{1,3,5}$ is nonempty.

2. $T_{1}(U)=\emptyset, T_{2}(U) \neq \emptyset$.

Based on $\left(M_{1,3,5}, \leq_{2}\right)$, the vertices $y_{1}, \ldots, y_{h}$ of $M_{1,3,5}$ can be totally ordered so that $y_{i}$ is maximal for $\left(\left\{y_{i}, \ldots, y_{h}\right\}, \leq_{2}\right)$ for $i=1, \ldots, h$. Then one can solve WIS in $G[U]$ by sequentially solving WIS in $G\left[U \backslash N\left(y_{1}\right)\right]$, in $\left.G\left[\left(U \backslash\left\{y_{1}, \ldots, y_{i-1}\right\}\right) \backslash N\left(y_{i}\right)\right)\right]$ for $i=2, \ldots, h$, and in $G\left[U \backslash M_{1,3,5}\right]$.

That can be done in $\mathcal{O}\left(n^{5}\right)$ time. In fact, let us first consider $G\left[U \backslash N\left(y_{1}\right)\right]$. If $G\left[\left(U \backslash N\left(y_{1}\right)\right)^{*}\right]$ admits no component of type 2, then one can refer to Case 1. Otherwise, by Lemma $4, G\left[\left(U \backslash N\left(y_{1}\right)\right)^{*}\right]$ admits a unique component of type 2, say $Q$. Then one can solve WIS in $G\left[U \backslash N\left(y_{1}\right)\right]$ by solving WIS in $G\left[\left(U \backslash N\left(y_{1}\right)\right) \backslash Q\right]$ and in $G\left[\left(U \backslash N\left(y_{1}\right)\right) \backslash N(q)\right]$, for every $q \in Q$ : since for each of such graphs $G[H]$ one has that $G\left[H^{*}\right]$ has no component of type 2, one can refer to Case 1 and to Lemmas 1 and 2. Now, let us consider $\left.G\left[\left(U \backslash\left\{y_{1}, \ldots, y_{i-1}\right\}\right) \backslash N\left(y_{i}\right)\right)\right]$ for $i=2, \ldots, h$ : by the mentioned total order, one can apply the argument applied for $G\left[U \backslash N\left(y_{1}\right)\right]$ in order to show that WIS can be 
solved for such graphs in polynomial time. Finally, let us consider $G\left[U \backslash M_{1,3,5}\right]$ : since no vertex in $U \backslash M_{1,3,5}$ is partial to any component of $G[Z(0)]$ (otherwise a $P_{6}$ arises), one can refer to Case 1.

3. $T_{1}(U) \neq \emptyset$.

Based on $\left(M_{1,3,5}, \leq_{1}\right)$, the vertices $y_{1}, \ldots, y_{h}$ of $M_{1,3,5}$ can be totally ordered so that $y_{i}$ is maximal for $\left(\left\{y_{i}, \ldots, y_{h}\right\}, \leq_{1}\right)$ for $i=1, \ldots, h$. Then one can solve WIS in $G[U]$ by sequentially solving WIS in $G\left[U \backslash N\left(y_{1}\right)\right]$, in $\left.G\left[\left(U \backslash\left\{y_{1}, \ldots, y_{i-1}\right\}\right) \backslash N\left(y_{i}\right)\right)\right]$ for $i=2, \ldots, h$, and in $G\left[U \backslash M_{1,3,5}\right]$.

That can be done in $\mathcal{O}\left(n^{6}\right)$ time. In fact, let us first consider $G\left[U \backslash N\left(y_{1}\right)\right]$. By Lemma $3, G\left[T_{1}(W) \backslash\right.$ $\left.N\left(y_{1}\right)\right]$ is $P_{3}$-free. Then $G\left[\left(U \backslash N\left(y_{1}\right)\right)^{*}\right]$ admits no component of type 1 . Then one can refer to Case 2 and to Lemmas 1 and 2 Now, let us consider $\left.G\left[\left(U \backslash\left\{y_{1}, \ldots, y_{i-1}\right\}\right) \backslash N\left(y_{i}\right)\right)\right]$ for $i=2, \ldots, h$ : by the mentioned total order, one can apply the argument applied for $G\left[U \backslash N\left(y_{1}\right)\right]$ in order to show that WIS can be solved for such graphs in polynomial time. Finally, let us consider $G\left[U \backslash M_{1,3,5}\right]$ : since no vertex in $U \backslash M_{1,3,5}$ is partial to any component of $G[Z(0)]$ (otherwise a $P_{6}$ arises), one can refer to Case 1

Let us summarize the above argument as follows.

Theorem 3 Let $G=(V, E)$ be a $\left(P_{6}\right.$,diamond)-free graph containing a 6-cycle $C$. Then one can solve WIS for $G[V \backslash N(c)]$ in $\mathcal{O}\left(n^{6}\right)$ time, for any vertex $c$ of $C$.

\subsection{Deleting $C_{5}$ 's in $\left(P_{6}\right.$, diamond, $\left.C_{6}\right)$-free graphs}

Throughout this subsection assume that $G=(V, E)$ is a $\left(P_{6}\right.$, diamond, $\left.C_{6}\right)$-free graph containing a 5-cycle $C$, say with vertices $v_{i}$ and edges $v_{i} v_{i+1}, i \in\{1, \ldots, 5\}$ (subscript addition taken modulo 5). Let $N(C)$ be the set of vertices from $V \backslash C$ which are adjacent to some vertex in $C$. For any subset $S$ of $C$, let $M_{S}$ be the set formed by those vertices in $N(C)$ which are adjacent to each vertex in $S$ and are nonadjacent to each vertex in $C \backslash S$. In particular, let us write $M_{1}$ for $S=\left\{v_{1}\right\}, M_{1,2}$ for $S=\left\{v_{1}, v_{2}\right\}$, and so on. Then let $Z(k)$ denote the set of vertices of $N(C)$ having exactly $k$ neighbors in $C$.

Since $G$ is $\left(P_{6}\right.$, diamond)-free: $Z(4)=Z(5)=\emptyset$; each element of $Z(3)$ belongs to some of the sets $M_{i, i+2, i+3}, i \in\{1, \ldots, 5\}$ (subscript addition taken modulo 5).

Similarly to the previous subsection, one has the following fact.

Lemma 5 Every component of $G[Z(0)]$ is green.

Lemma 6 There exists a vertex $c$ of $C$ such that one of the following statements holds:

(i) $M_{i} \backslash N(c)=\emptyset$ for all $i \in\{2, \ldots, 5\}$, and $M_{i, i+1} \backslash N(c)=\emptyset$ for all $i \in\{1, \ldots, 5\}$ (subscript addition taken modulo 5);

(ii) $M_{i} \backslash N(c)=\emptyset$ for all $i \in\{1, \ldots, 5\}$, and $M_{i, i+1} \backslash N(c)=\emptyset$ for all $i \in\{1, \ldots, 4\}$ (subscript addition taken modulo 5).

Proof: Since $G$ is $\left(P_{6}, C_{6}\right)$-free, for all $i \in\{1, \ldots, 5\}$ (subscript addition taken modulo 5) one has that: if $M_{i} \neq \emptyset$, then $M_{i+2}=M_{i+3}=M_{i+1, i+2}=M_{i+3, i+4}=\emptyset$; if $M_{i, i+1} \neq \emptyset$, then $M_{i-1, i}=M_{i+1, i+2}=$ $\emptyset$. This implies the lemma.

Let us fix any vertex of $C$, say $v_{2}$, guaranteed by Lemma 6 . 
Let us prove that one can solve WIS for $G\left[V \backslash N\left(v_{2}\right)\right]$ in $\mathcal{O}\left(n^{4}\right)$ time.

A partition of $V \backslash N\left(v_{2}\right)$ is given by $\left\{\left\{v_{2}, v_{4}, v_{5}\right\}, M_{1,3,4}, M_{3,4,5}, M_{1,3}, M_{1,4}, M_{3,5}, M_{1}, Z(0)\right\}$.

Since $G$ is diamond-free, the sets $M_{1,3,4}, M_{3,4,5}$ have cardinality at most one. Then, by Observation 1 . to our aim it is sufficient to prove that WIS can be solved for each induced subgraph of $G[U]$ in polynomial time, where a partition of $U$ is given by $\left\{M_{1,3}, M_{1,4}, M_{3,5}, M_{1}, Z(0)\right\}$.

Since $G$ is diamond-free, $M_{1,3}, M_{1,4}, M_{3,5}$ are independent sets. Since $G$ is $P_{6}$-free: $M_{1}$ has a co-join with $Z(0)$; each vertex in $M_{1,3} \cup M_{1,4} \cup M_{3,5}$ is not partial to any component of $G[Z(0)]$, i.e., each component of $G[Z(0)]$ is a module of $G[U]$. So by assertions similar to Lemmas 5 and 1 . one can assume that $Z(0)$ is an independent set.

Now, let us consider the following cases, which are exhaustive by symmetry.

Case 1 statement还of Lemma 6 holds.

Case 1.1 $M_{1}=\emptyset$. One can solve WIS in $G[U]$ by solving WIS in $G\left[U \backslash M_{3,5}\right]$ and in $G[U \backslash N(y)]$ for every $y \in M_{3,5}$. Since $G$ is diamond-free, one can verify that such graphs are triangle-free. Then in this case one can solve WIS for $G[U]$ in $\mathcal{O}\left(n^{3}\right)$ time by Theorem 2

Case 1.2 $M_{1} \neq \emptyset$.

First assume that $M_{1}$ is a clique. One can solve WIS in $G[U]$ by solving WIS in $G\left[U \backslash M_{1}\right]$ and in $G[U \backslash N(y)]$ for every $y \in M_{1}$. Then, by referring to Case 1.1, in this case one can solve WIS for $G[U]$ in $\mathcal{O}\left(n^{4}\right)$ time.

Then assume that $M_{1}$ is not a clique, i.e., $G\left[M_{1}\right]$ is disconnected (since $G\left[M_{1}\right]$ is $P_{3}$-free). Then $M_{3,5}$ is partitioned into $\left\{M_{3,5}^{0}, M_{3,5}^{\prime}\right\}$, where $M_{3,5}^{\prime}=\left\{x \in M_{3,5}: x\right.$ is universal for $\left.M_{1}\right\}$, and $M_{3,5}^{0}=\left\{x \in M_{3,5}: x\right.$ does not contact $\left.M_{1}\right\}$ (in fact if $y, z \in M_{1}$ are nonadjacent and a vertex $x \in M_{3,5}$ is adjacent to $y$ and nonadjacent to $z$, then $v_{4}, v_{3}, x, y, v_{1}, z$ induce a $P_{6}$ ). One can solve WIS in $G[U]$ by solving WIS in $G\left[U \backslash M_{3,5}^{\prime}\right]$ and in $G[U \backslash N(y)]$ for every $y \in M_{3,5}^{\prime}$.

That can be done in $\mathcal{O}\left(n^{4}\right)$ time. Concerning graphs $G[U \backslash N(y)]$ for every $y \in M_{3,5}^{\prime}$, one can refer to Case 1.1. Then let us consider $G\left[U \backslash M_{3,5}^{\prime}\right]$. Notice that $M_{3,5}^{0}$ has a co-join with $Z(0)$, otherwise a $P_{6}$ arises involving a vertex of $M_{1}$. Then $U \backslash M_{3,5}^{\prime}$ is partitioned into $\{X, Y\}$, where $X=M_{1} \cup M_{1,3} \cup M_{1,4}$ (i.e., $G[X]$ is $P_{3}$-free) and $Y=M_{3,5}^{0} \cup Z(0)$ (i.e., $Y$ is an independent set). Then each component of $G\left[U \backslash M_{3,5}^{\prime}\right]$ is either $P_{3}$-free or green. Then the assertion follows by Lemma[1.

Case 2 statement iil of Lemma 6 holds.

One can solve WIS in $G[U]$ by solving WIS in $G\left[U \backslash M_{4,5}\right]$ and in $G[U \backslash N(y)]$ for every $y \in M_{4,5}$. Since $G$ is diamond-free, $M_{4,5}$ is a clique. Then, by referring to Case 1.1 (3.2), in this case one can solve WIS for $G[U]$ in $\mathcal{O}\left(n^{4}\right)$ time.

Let us summarize the above argument as follows.

Theorem 4 Let $G=(V, E)$ be a $\left(P_{6}\right.$,diamond, $\left.C_{6}\right)$-free graph containing a 5-cycle $C$.

Then there exists a vertex cof $C$ (which can be easily found) such that one can solve WIS for $G[V \backslash N(c)]$ in $\mathcal{O}\left(n^{4}\right)$ time. 


\subsection{Deleting $C_{4}$ 's in $\left(P_{6}\right.$, diamond, $\left.C_{6}, C_{5}\right)$-free graphs}

Throughout this subsection assume that $G=(V, E)$ is a $\left(P_{6}\right.$,diamond, $\left.C_{6}, C_{5}\right)$-free graph containing a 4-cycle $C$, say with vertices $v_{i}$ and edges $v_{i} v_{i+1}, i \in\{1, \ldots, 4\}$ (subscript addition taken modulo 4). Let $N(C)$ be the set of vertices from $V \backslash C$ which are adjacent to some vertex in $C$. For any subset $S$ of $C$, let $M_{S}$ be the set formed by those vertices in $N(C)$ which are adjacent to each vertex in $S$ and are nonadjacent to each vertex in $C \backslash S$. In particular, let us write $M_{1}$ for $S=\left\{v_{1}\right\}, M_{1,2}$ for $S=\left\{v_{1}, v_{2}\right\}$, and so on. Then let $Z(k)$ denote the set of vertices of $N(C)$ having exactly $k$ neighbors in $C$.

Since $G$ is $\left(P_{6}\right.$, diamond)-free: $Z(3)=Z(4)=\emptyset$.

Similarly to the previous subsection, one has the following fact.

Lemma 7 Every component of $G[Z(0)]$ is green.

Let us fix any vertex of $C$, say $v_{2}$.

Let us prove that WIS can be solved for $G\left[V \backslash N\left(v_{2}\right)\right]$ in $\mathcal{O}\left(n^{6}\right)$ time.

A partition of $V \backslash N\left(v_{2}\right)$ is given by $\left\{\left\{v_{2}, v_{4}\right\}, M_{1,3}, M_{3,4}, M_{4,1}, M_{1}, M_{3}, M_{4}, Z(0)\right\}$. Then, by Observation 1 to our aim it is sufficient to prove that WIS can be solved for each induced subgraph of $G[U]$ in polynomial time, where a partition of $U$ is given by $\left\{M_{1,3}, M_{3,4}, M_{4,1}, M_{1}, M_{3}, M_{4}, Z(0)\right\}$,

Let us introduce some preliminary definitions and lemmas.

Let us write:

$$
\begin{aligned}
& M_{1}^{0}=\left\{x \in M_{1}: x \text { does not contact } M_{4}\right\} \\
& M_{3}^{0}=\left\{x \in M_{3}: x \text { does not contact } M_{4}\right\} \\
& M_{4}^{0}=\left\{x \in M_{4}: x \text { does not contact } M_{1} \cup M_{3}\right\} \\
& X=\left\{x \in M_{1}: x \text { contacts } M_{4}\right\} \cup\left\{x \in M_{4}: x \text { contacts } M_{1}\right\} \\
& Y=\left\{x \in M_{3}: x \text { contacts } M_{4}\right\} \cup\left\{x \in M_{4}: x \text { contacts } M_{3}\right\}
\end{aligned}
$$

Let us write:

$$
\begin{aligned}
Z_{1} & =M_{1}^{0} \cup\left\{z \in Z(0): z \text { contacts } M_{1}^{0}\right\} \\
Z_{3} & =M_{3}^{0} \cup\left\{z \in Z(0): z \text { contacts } M_{3}^{0}\right\} \\
Z_{4} & =M_{4}^{0} \cup\left\{z \in Z(0): z \text { contacts } M_{4}^{0}\right\} \\
Z_{X} & =X \cup\{z \in Z(0): z \text { contacts } X\} \\
Z_{Y} & =Y \cup\{z \in Z(0): z \text { contacts } Y\} \\
\tilde{Z} & =\left\{z \in Z(0): z \text { does not contact } M_{1} \cup M_{3} \cup M_{4}\right\}
\end{aligned}
$$

Lemma 8 The following facts hold:

(i) each pair of the sets $Z_{1}, Z_{3}, Z_{4}, Z_{X}, Z_{Y}, \tilde{Z}$ has a co-join;

(ii) each component of $G\left[Z_{1} \cup Z_{3} \cup Z_{4} \cup \tilde{Z}\right]$ is green;

(iii) each component of $G\left[Z_{X} \cup Z_{Y}\right]$ is either $P_{3}$-free or bipartite.

\section{Proof:}


Proof of it Since $G$ is $C_{5}$-free, $M_{1}$ has a co-join with $M_{3}$. Then, since $G$ is $C_{6}$-free, no vertex in $M_{4}$ can be adjacent to a vertex in $M_{1}$ and to a vertex in $M_{3}$ at the same time. It follows that each pair of the sets $M_{1}^{0}, M_{3}^{0}, M_{4}^{0}, X, Y$ has a co-join. Furthermore, since $G$ is $\left(P_{6}, C_{6}\right)$-free, one can verify that two vertices chosen in two different sets - among the mentioned sets - cannot contact a component of $Z(0)$ at the same time. Finally, since $G$ is $P_{6}$-free, if a vertex in $y \in M_{1} \cup M_{3} \cup M_{4}$ contacts a component $K$ of $G[Z(0)]$, then $y$ is universal for $K$. Then if follows.

Proof of iii It is enough to deal with $G\left[Z_{1}\right]$, as the other subsets can be treated similarly. If $Z_{1}=M_{1}^{0}$, then $G\left[Z_{1}\right]$ is $P_{3}$-free. Otherwise, since $G$ is ( $P_{6}$,diamond)-free, one can verify (similarly to the argument of Lemma 2 that each component of $G\left[Z_{1}\right]$ is green.

Proof of iii. It is enough to deal with $G\left[Z_{1}\right]$, as the other subsets can be treated similarly. If $Z_{X}=X$, then since $G$ is (diamond, $C_{5}$ )-free, each component of $G\left[Z_{X}\right]$ is bipartite. Otherwise, since $G$ is $P_{6}$-free, each vertex in $Z(0)$ contacting a component $K$ of $G[X]$ dominates $K$. Then, since $G$ is diamond-free, each component of $G\left[Z_{X}\right]$ is a clique.

Lemma 9 If $M_{1} \neq \emptyset$ and $M_{3} \neq \emptyset$, then:

(i) $Z(0)$ has a co-join with $M_{1} \cup M_{3}$;

(ii) $X=Y=\emptyset$.

\section{Proof:}

Proof of it It follows since $G$ is $\left(P_{6}, C_{6}, C_{5}\right)$-free.

Proof of iif. By symmetry, let us only prove that $X=\emptyset$. Assume for a contradiction that $X \neq \emptyset$. Then let $x_{1} \in M_{1}$ be adjacent to $x_{4} \in M_{4}$. Let $x_{3} \in M_{3}$. By 1 i of Lemma 8 , $x_{1}$ and $x_{4}$ are nonadjacent to $x_{3}$. Then $x_{4}, x_{1}, v_{1}, v_{2}, v_{3}, x_{3}$ induce a $P_{6}$.

Lemma 10 If a vertex $y \in M_{1,3}$ contacts a component $K$ of $G\left[Z_{X} \cup Z_{Y}\right]$, then $K \backslash N(y)$ is either a clique or an independent set.

Proof: By symmetry, let us consider only $G\left[Z_{X}\right]$. Let $K$ be a component of $G[X]$. If $G[K]$ is $P_{3}$-free, then the assertion trivially follows. Then, by iiii of Lemma 8 , assume that $G[K]$ is bipartite. Let $y \in M_{1,3}$ contact $K$. Notice that $y$ cannot be adjacent to two adjacent vertices of $K$, otherwise a diamond arises involving $v_{1}$. Then, to avoid a $P_{6}, y$ is adjacent to all the vertices of a side of the bipartite graph, i.e., $K \backslash N(y)$ is an independent set.

Let us write $Z=Z_{1} \cup Z_{3} \cup Z_{4} \cup Z_{X} \cup Z_{Y} \cup \tilde{Z}$. Then $\left\{M_{1,3}, Z\right\}$ is a partition of $U$.

For any $W \subseteq U$, let us write $W^{*}=W \cap Z$.

For any $W \subseteq U$, let us say that a component $K$ of $G\left[W^{*}\right]$ is of:

type 1 if $K$ is not a clique and there exists a vertex in $W \backslash Z$ partial to $K$;

type 2 if $K$ is a clique and there exists a vertex in $W \backslash Z$ partial to $K$;

type 3 otherwise. 
Let $T_{1}(W), T_{2}(W), T_{3}(W)$ respectively denote the union of components of $G\left[W^{*}\right]$ of type $1,2,3$.

Let us fix a subset $W \subseteq U$.

Notice that $M_{1,3}$ is an independent set, since $G$ is diamond-free.

Let us consider the following binary relation ' $\leq_{1}$ ' on $M_{1,3}$ : for any $a, b \in M_{1,3}, a \leq_{1} b$ if $N_{T_{1}(W)}(a) \subseteq$ $N_{T_{1}(W)}(b)$. It is immediate to verify that $\left(M_{1,3}, \leq_{1}\right)$ is a partially ordered set.

Lemma 11 Let $y \in M_{1,3}$ be maximal for $\left(M_{1,3}, \leq_{1}\right)$. Then $G\left[T_{1}(W) \backslash N(y)\right]$ is $P_{3}$-free.

Proof: If either $M_{1}=\emptyset$ or $M_{3}=\emptyset$, then one can apply an argument similar to that of Lemma 3 , by considering also $(i)$ of Lemma 8 and Lemma 10 - in detail, if $M_{1}=\emptyset$ (if $M_{3}=\emptyset$ ), then vertex $v_{1}$ (vertex $\left.v_{3}\right)$ is universal for $M_{1,3}$ and does not contact $Z$.

If $M_{1} \neq \emptyset$ and $M_{3} \neq \emptyset$, then one can apply an argument similar to that of Lemma 3 , by considering Lemma 9 and the fact that no element of $M_{1,3}$ can be partial to a component of $G\left[M_{1} \cup M_{3}\right]$ (since $G$ is diamond-free).

Let us consider the following binary relation ' $\leq_{2}$ ' on $M_{1,3}$ : for any $a, b \in M_{1,3}, a \leq_{2} b$ if $N_{T_{2}(W)}(a) \subseteq$ $N_{T_{2}(W)}(b)$. It is immediate to verify that $\left(M_{1,3}, \leq_{2}\right)$ is a partially ordered set.

Lemma 12 Let $y \in M_{1,3}$ be maximal for $\left(M_{1,3}, \leq_{2}\right)$. If $T_{1}(W)=\emptyset$, then $G\left[(W \backslash N(y))^{*}\right]$ admits at most one component of type 2 .

Proof: One can apply the argument in the proof of Lemma 11, by considering Lemma 4 instead of Lemma 3 .

Now, let us consider the following cases.

Case $1 M_{3,4}=M_{4,1}=\emptyset$.

Case 1.1 $T_{1}(U)=T_{2}(U)=\emptyset$.

Then each component $K$ of $G[Z]$ is a module of $G[U]$. Then, to our aim, one can assume that $K$ is a singleton. In fact, one can contract $K$ into a singleton $k$ with $w(k)=\alpha_{w}(G[K])$ : that can be done in $\mathcal{O}\left(n^{3}\right)$ time by ii- iii of Lemma 8 and Lemma 1 .

So in general, one can assume that $Z$ is an independent set. Then $G[U]$ is bipartite. In this case one can solve WIS for $G[U]$ in time $\mathcal{O}\left(n^{3}\right)$ by Theorem 2

Case $1.2 T_{1}(U)=\emptyset, T_{2}(U) \neq \emptyset$.

Based on $\left(M_{1,3}, \leq_{2}\right)$, the vertices $y_{1}, \ldots, y_{h}$ of $M_{1,3}$ can be totally ordered so that $y_{i}$ is maximal for $\left(\left\{y_{i}, \ldots, y_{h}\right\}, \leq_{2}\right)$ for $i=1, \ldots, h$. Then one can solve WIS in $G[U]$ by sequentially solving WIS in $G\left[U \backslash N\left(y_{1}\right)\right]$, in $\left.G\left[\left(U \backslash\left\{y_{1}, \ldots, y_{i-1}\right\}\right) \backslash N\left(y_{i}\right)\right)\right]$ for $i=2, \ldots, h$, and in $G\left[U \backslash M_{1,3}\right]$.

That can be done in $\mathcal{O}\left(n^{5}\right)$ time. In fact, let us first consider $G\left[U \backslash N\left(y_{1}\right)\right]$. If $G\left[\left(U \backslash N\left(y_{1}\right)\right)^{*}\right]$ admits no component of type 2 , then one can refer to Case 1.1. Otherwise, by Lemma $12, G[(U \backslash$ $\left.\left.N\left(y_{1}\right)\right)^{*}\right]$ admits a unique component of type 2, say $Q$. Then one can solve WIS in $G\left[U \backslash N\left(y_{1}\right)\right]$ by solving WIS in $\left.G\left[\left(U \backslash N\left(y_{1}\right)\right) \backslash Q\right)\right]$ and in $G\left[\left(U \backslash N\left(y_{1}\right)\right) \backslash N(q)\right]$, for every $q \in Q$ : since for each of such graphs $G[H]$ one has that $G\left[H^{*}\right]$ has no component of type 2, one can refer to Case 1.1, to ii iiii of Lemma 8 and to Lemmas 7 and 1 . Now, let us consider $\left.G\left[\left(U \backslash\left\{y_{1}, \ldots, y_{i-1}\right\}\right) \backslash N\left(y_{i}\right)\right)\right]$ for $i=2, \ldots, h$ : by the mentioned total order, one can apply the argument applied for $G\left[U \backslash N\left(y_{1}\right)\right]$ in 
order to show that WIS can be solved for such graphs in polynomial time. Finally, let us consider $G\left[U \backslash M_{1,3}\right]=G[Z]$ : then one can refer to Case 1.1.

Case 1.3 $T_{1}(U) \neq \emptyset$.

Based on $\left(M_{1,3}, \leq_{2}\right)$, the vertices $y_{1}, \ldots, y_{h}$ of $M_{1,3}$ can be totally ordered so that $y_{i}$ is maximal for $\left(\left\{y_{i}, \ldots, y_{h}\right\}, \leq_{2}\right)$ for $i=1, \ldots, h$. Then one can solve WIS in $G[U]$ by sequentially solving WIS in $G\left[U \backslash N\left(y_{1}\right)\right]$, in $\left.G\left[\left(U \backslash\left\{y_{1}, \ldots, y_{i-1}\right\}\right) \backslash N\left(y_{i}\right)\right)\right]$ for $i=2, \ldots, h$, and in $G\left[U \backslash M_{1,3}\right]$.

That can be done in $\mathcal{O}\left(n^{6}\right)$ time. In fact, let us first consider $G\left[U \backslash N\left(y_{1}\right)\right]$. By Lemma 11 . $G\left[T_{1}(W) \backslash N\left(y_{1}\right)\right]$ is $P_{3}$-free. Then $G\left[\left(U \backslash N\left(y_{1}\right)\right)^{*}\right]$ admits no component of type 1 . Then one can refer to Case 1.2 and to Lemmas 7 and 1 Now, let us consider $\left.G\left[\left(U \backslash\left\{y_{1}, \ldots, y_{i-1}\right\}\right) \backslash N\left(y_{i}\right)\right)\right]$ for $i=2, \ldots, h$ : by the total order, one can apply the argument applied for $G\left[U \backslash N\left(y_{1}\right)\right]$ in order to show that WIS can be solved for such graphs in polynomial time. Finally, let us consider $G\left[U \backslash M_{1,3}\right]=G[Z]$ : then one can refer to Case 1.1.

Case $2 M_{3,4} \cup M_{4,1} \neq \emptyset$.

Since $G$ is diamond-free, $M_{3,4}$ and $M_{4,1}$ are cliques. Since $G$ is ( $P_{6}$,diamond, $C_{6}, C_{5}$ )-free, the following facts hold: $M_{3,4} \cup M_{4,1}$ has a co-join with $N(C) \backslash\left(N\left(v_{2}\right) \cup M_{3,4} \cup M_{4,1}\right)$; a vertex in $M_{3,4} \cup M_{4,1}$ and a vertex in $N(C) \backslash\left(N\left(v_{2}\right) \cup M_{3,4} \cup M_{4,1}\right)$ cannot contact a component of $G[Z(0)]$ at the same time; $M_{3,4}$ has a co-join with $M_{4,1}$.

First assume that $M_{3,4} \neq \emptyset$ and $M_{4,1} \neq \emptyset$. Then $M_{3,4} \cup M_{4,1}$ has a co-join with $Z(0)$ (otherwise a $P_{6}$ or a $C_{6}$ arises). In general, by the above facts, $M_{3,4} \cup M_{4,1}$ has a co-join with $U \backslash\left(M_{3,4} \cup M_{4,1}\right)$. Then, since $M_{3,4}$ and $M_{4,1}$ are cliques, one can directly refer to Case 1.

Then assume that $M_{3,4}=\emptyset$. One can solve WIS for $G[U]$ by solving WIS in $G\left[U \backslash M_{4,1}\right]$ and in $G[U \backslash N(y)]$, for every $y \in M_{4,1}$. Since $M_{4,1}$ is a clique, one can directly refer to Case 1 .

The case in which $M_{4,1}=\emptyset$ can be similarly treated, by symmetry.

Let us summarize the above argument as follows.

Theorem 5 Let $G=(V, E)$ be a $\left(P_{6}\right.$,diamond, $\left.C_{6}, C_{5}\right)$-free graph containing a 4-cycle $C$. Then one can solve WIS for $G[V \backslash N(c)]$ in $\mathcal{O}\left(n^{6}\right)$ time, for any vertex $c$ of $C$.

\subsection{A solution for WIS and WID in ( $P_{6}$, diamond)-free graphs}

In this subsection we formalize an efficient method for solving WIS (or WID) in ( $P_{6}$, diamond)-free graphs. To this end, let us first summarize the results of the previous subsections in the following theorem.

Theorem 6 Let $G=(V, E)$ be a connected ( $P_{6}$,diamond)-free graph containing a $C_{6}$ or a $C_{5}$ or a $C_{4}$. Then there exists a vertex c (which can be easily found) such that one can solve WIS (or WID) for $G[V \backslash N(c)]$ in $\mathcal{O}\left(n^{6}\right)$ time.

Proof: If $G$ contains a $C_{6}$, then the assertion follows by Theorem 3 . If $G$ is $C_{6}$-free and contains a $C_{5}$, then the assertion follows by Theorem 4 If $G$ is $\left(C_{6}, C_{5}\right)$-free and contains a $C_{4}$, then the assertion follows by Theorem 5 .

To prove that WIS (or WID) is solvable in polynomial time on the class of ( $P_{6}$,diamond)-free graphs, it suffices to find a polynomial upper bound $p(n)=O\left(n^{7}\right)$ on the number of steps sufficient for any 
allowed input of order $n$. If $G$ is chordal, then we are done by Theorem 1 Otherwise, there exists a sixth-degree polynomial $q(n)$ with the property that in any ( $P_{6}$,diamond)-free non-chordal graph one can determine a vertex $x$ such that WIS (or WID) can be solved on $G_{1}=G[V \backslash N(x)]$ in $q(n)$ time. If $G_{1}^{\prime}=G-x$ is not chordal, then again one can find a vertex $x^{\prime}$ such that the problem can be solved on $G_{2}=G\left[V\left(G_{1}^{\prime}\right) \backslash N\left(x^{\prime}\right)\right]$ in $q(n)$ time, and so on. In this way we obtain some graphs $G_{1}, G_{2}, \ldots, G_{k}$ with $k<n$, such that each $G_{i}$ is either chordal or admits an efficient WIS (or WID) algorithm. Thus, the total running time is $O(n(q(n)+r(n)))$ where $r(n)$ is the time needed to check whether the current graph is chordal and if it is not, then to find a suitable vertex under the conditions of Theorem 6 .

Now, by Theorems 1 and 6 one obtains:

Theorem 7 Both the WIS and the WID problems can be solved for ( $P_{6}$,diamond)-free graphs in $\mathcal{O}\left(n^{7}\right)$ time.

\section{Acknowledgements}

I would like to thank the referees for their valuable remarks and suggestions, and the handling editor for his helpful and resolving comments. 


\section{References}

[1] V.E. Alekseev, On the local restriction effect on the complexity of finding the graph independence number, Combinatorial-algebraic Methods in Applied Mathematics, Gorkiy University Press, Gorkiy (1983) 3-13 (in Russian)

[2] V.E. Alekseev, A polynomial algorithm for finding largest independent sets in fork-free graphs, Discrete Analysis and Operations Research Ser. 1, 6 (1999) 3-19 (in Russian), Discrete Applied Mathematics 135 (2004) 3-16

[3] V.E. Alekseev, On easy and hard hereditary classes of graphs with respect to the independent set problem, Discrete Applied Mathematics 132 (2004) 17-26

[4] C. Arbib, R. Mosca, On ( $P_{5}$,diamond)-free graphs, Discrete Mathematics 250 (2002) 1-22

[5] G. Bacsó, Zs. Tuza, Dominating cliques in $P_{5}$-free graphs, Periodica Mathematica Hungarica 21, 4 (1990) 303-308

[6] G. Bacsó, Zs. Tuza, A characterization of graphs without long induced paths, J. Graph Theory 14, 4 (1990) 455-464

[7] R. Boliac, V.V. Lozin, Independent domination in finitely defined classes of graphs, Theoretical Computer Science 301 (2003) 271-284

[8] A. Brandstädt, $\left(P_{5}\right.$,diamond)-free graphs revisited: structure and linear time optimization, Discrete Applied Mathematics 138 (2004) 13-27

[9] A. Brandstädt, Chính T. Hoàng, On clique separators, nearly chordal graphs and the Maximum Weight Stable Set problem, M. Jünger and V. Kaibel (Eds.): IPCO 2005, LNCS 3509, pp. 265-275, 2005

[10] A. Brandstädt, H.-O. Le, V.B. Le, On $\alpha$-redundant vertices in $P_{5}$-free graphs, Information Processing Letters 82 (2002) 119-122

[11] A. Brandstädt, V.B. Le, S. Mahfud, New applications of clique separators decomposition for the Maximum Weight Stable Set problem, Theoretical Computer Science 370 (2007) 229-239

[12] A. Brandstädt, V.B. Le, J.P. Spinrad, Graph Classes: A Survey, SIAM Monographs on Discrete Math. Appl., Vol. 3, SIAM, Philadelphia (1999)

[13] A. Brandstädt, V.V. Lozin, A note on $\alpha$-redundant vertices in graphs, Discrete Applied Mathematics 108 (2001) 301-308

[14] A. Brandstädt, T. Klembt, S. Mahfud, $P_{6^{-}}$and Triangle-Free Graphs Revisited: Structure and Bounded Clique-Width, Discrete Mathematics and Theoretical Computer Science 8 (2006) 173-188

[15] A. Brandstädt, D. Kratsch, On domination problems on permutation and other graphs, Theoretical Computer Science 54 (1987) 181-198 
[16] A. Brandstädt, D. Kratsch, On the structure of $\left(P_{5}\right.$, gem)-free graphs, Dicrete Applied Mathematics 145 (2005) 155-166

[17] A. Brandstädt, R. Mosca, On the structure and stability number of $P_{5^{-}}$and co-chair-free graphs, Discrete Applied Mathematics 132 (2004) 47-65

[18] G. Chang, The weighted independent domination problem is NP-complete for chordal graphs, Discrete Applied Mathematics 143 (2004) 351-352

[19] J. Dong, On the $i$-diameter of $i$-center in a graph without long induced paths, $J$. Graph Theory 30 (1999) 235-241

[20] M. Farber, Independent domination in chordal graphs, Operations Reseach Letters 1 (1982) 134-138

[21] M. Farber, On diameters and radii of bridged graphs, Discrete Mathematics 73 (1989) 249-260

[22] M. Farber, M. Hujter, Zs. Tuza, An upper bound on the number of cliques in a graph, Networks 23 (1993) $75-83$

[23] J.-L. Fouquet, V. Giakoumakis, F. Maire, H. Thuillier, On graphs without $P_{5}$ and $\bar{P}_{5}$, Discrete Mathematics 146 (1995) 33-44

[24] J.-L. Fouquet, V. Giakoumakis, J.-M. Vanherpe, Bipartite graphs totally decomposable by canonical decomposition, International Journal of Foundations of Computer Science 10 (1999) 513-533

[25] A. Frank, Some polynomial algorithms for certain graphs and hypergraphs, Proceedings of the Fifth British Combinatorial Conference (Univ. Aberdeen, Aberdeen 1975) 211-226; Congressus Numerantium No. XV, Utilitas Math., Winnipeg, Man. (1976)

[26] M.R. Garey, D.S. Johnson, L. Stockmeyer, Some simplified NP-complete graph problems, Theoretical Computer Science 1 (1976) 237-267

[27] M.R. Garey, D.S. Johnson, The rectilinear Steiner tree problem is NP-complete, SIAM J. Applied Mathematics 32 (1977) 826-834

[28] M.R. Garey, D.S. Johnson, Computers and Intractability: A Guide to the Theory of NP-completness, Freeman, San Francisco, CA (1979)

[29] M. Gerber, V.V. Lozin, On the stable set problem in special $P_{5}$-free graphs, Discrete Applied Mathematics 125 (2003) 215-224

[30] V. Giakoumakis, J.-M. Vanherpe, Linear time recognition and optimization for weak-bisplit graphs, bi-cographs and bipartite $P_{6}$-free graphs, International Journal of Foundations of Computer Science 14 (2003)107-136

[31] V. Giakoumakis, I. Rusu, Weighted parameters in $\left(P_{5}, \bar{P}_{5}\right)$-free graphs, Discrete Applied Mathematics 80 (1997) 255-261

[32] M.C. Golumbic, U. Rotics, On the clique-width of some perfect graph classes, International Journal of Foundations of Computer Science 11 (2000) 423-443 
[33] M. Grötschel, L. Lovász, A. Schrijver, Polynomial algorithms for perfect graphs, Annals of Discrete Mathematics 21 (1984) 325-356

[34] R.M. Karp, Reducibility among combinatorial problems, Complexity of Computer Computations, R.E. Miller, J.W. Thatcher (eds.), Plenum Press, New York (1972) 85-103

[35] J. Liu, H. Zhou, Dominating subgraphs in graphs with some forbidden structure, Discrete Mathematics 135 (1994) 163-168

[36] V.V. Lozin, Stability in $P_{5^{-}}$and banner-free graphs, European J. Operational Research 125 (2000) 292-297

[37] V.V. Lozin, M. Milanič, A polynomial algorithm to find an independent set of maximum weight in a fork-free graph, RUTCOR Research Report, Rutgers University, 30-2005

[38] V.V. Lozin, R. Mosca, Independent sets in extensions of $2 K_{2}$-free graphs, Discrete Applied Mathematics 146 (2005) 74-80

[39] V.V. Lozin, D. Rautenbach, Some results on graphs without long induced paths, Information Processing Letters 88 (2003) 167-171

[40] G.J. Minty, On maximal independent sets of vertices in claw-free graphs, J. Combinatorial Theory, Series B 28 (1980) 284-304

[41] R. Mosca, Stable sets in certain $P_{6}$-free graphs, Discrete Applied Mathematics 92 (1999) 177-191

[42] D. Nakamura, A. Tamura, A revision of Minty's algorithm for finding a maximum weight stable set in a claw-free graph, J. Operations Research Society of Japan 44 (2001) 194-204

[43] S. Poljak, A note on stable sets and colorings of graphs, Commun. Math. Univ. Carolinae 15 (1974) 307-309

[44] B. Randerath, I. Schiermeyer, M. Tewes, Three-colourability and forbidden subgraphs. II: polynomial algorithms, Discrete Mathematics 251 (2002) 137-153

[45] N. Sbihi, Algorithme de recherche d'un stable de cardinalité maximum dans un graphe sans étoile, Discrete Mathematics 29 (1980) 53-76

[46] I.E. Zverovich, Independent domination on $2 P_{3}$-free perfect graphs, DIMACS Technical Report 2003-22, Rutgers University, 2003

[47] I.E. Zverovich, V.E. Zverovich, Locally well-dominated and locally independent well-dominated graphs, Graphs and Combinatorics 19 (2) (2003) 279-288 